\title{
Duality in Disk Induced Flows
}

\author{
Joachim Giesen and Matthias John \\ Institute for Theoretical Computer Science, ETH Zürich, CH-8092 Zürich \\ \{giesen, john\}@inf .ethz.ch
}

\begin{abstract}
We introduce a condition that establishes a duality known from Delaunay triangulations and Voronoi diagrams for diagrams associated with a class of dynamical systems defined via a set of disks in the plane. Under this condition the maximum geometric and worst case algorithmic complexities of the latter diagrams decrease. The condition is natural in the sense that it is automatically fulfilled by some important classes of sets of disks in the plane.
\end{abstract}

Keywords. Computational geometry, Voronoi diagram, Delaunay triangulation

\section{Introduction}

It is possible to associate a dynamical system with a set of disks in the plane which we want to call disk induced flows. In [4,5] we studied the fixpoints of these dynamical systems, i.e. the minima, maxima and saddles of the flow. With a minimum $m$ we associate all points that are connected to $m$ by the disk induced flow, i.e. all points $x$ that are connected to $m$ by a curve such that all points in the interior of the curve flow into $x$ under the disk induced flow. The set of all these points is a connected region in the plane that we call the Min region of $m$. Similarly Max regions are assigned to maxima. The collection of all Min regions defines a plane diagram that is closely related to the Delaunay triangulation of the same set of disks. The analogous diagram of the Max regions is closely related to the Voronoi diagram. The Delaunay diagram of a set of disks is the Voronoi diagram of a dual set of disks and vice versa. This is not the case for Min- and Max diagrams, i.e. the Min diagram of a set of disks need not be the Max diagram of the dual set of disks or vice versa. That is, in general a set of disks in the plane gives rise to four different flow diagrams, namely the Minand Max diagrams of the original and the dual set of disks. There are further differences between Delaunay- and Min diagrams or Voronoi- and Max diagrams, respectively. The maximum geometric complexities of Min- and Max diagrams associated with $n$ disks are $\Theta\left(n^{2}\right)$ vertices, $\Theta\left(n^{2}\right)$ edges and $\Theta(n)$ regions. This is in contrast to Voronoi- and Delaunay diagrams whose geometric complexities are: $\Theta(n)$ vertices, $\Theta(n)$ edges and $\Theta(n)$ regions. The worst case algorithmic

\footnotetext{
* Partly supported by the IST Programme of the EU as a Shared-cost RTD (FET Open) Project under Contract No IST-2000-26473 (ECG - Effective Computational Geometry for Curves and Surfaces).
} 
complexity of Min- and Max diagrams is $\Theta\left(n^{2}\right)$ in contrast to the worst case algorithmic complexity of Voronoi- and Delaunay diagrams which is $\Theta(n \log n)$.

Here we introduce a natural condition under which one of the dualities between Voronoi- and Delaunay diagrams also holds for Min- and Max diagrams. This condition is for example always fulfilled for finite sets of points, i.e. disks of radius zero. We call the condition strong duality condition since it has some strong implications. In fact we will show that under the strong duality condition the following holds:

(1) The Max diagram of the original set of disks is the Min diagram of the dual set of disks.

(2) The Min diagram of the original set of disks is the Voronoi diagram of these disks.

(3) The maximum geometric complexities of the Min- and Max diagram of the original set of disk are $\Theta(n)$ vertices, $\Theta(n)$ edges and $\Theta(n)$ regions.

(4) The worst case algorithmic complexity the Min- and Max diagram of the original set of disk are $\Theta(n \log n)$.

(5) The Max diagram of the original set of disks coincides with a diagram associated with a discrete flow on the set of Delaunay triangles. This discrete flow was studied in [3] to identify and compute pockets in macromolecules.

The paper is organized as follows: In Section 2 we give basic definitions. In Section 3 we introduce disk induced flows and the associated notions of Maxand Min regions. Finally we introduce the strong duality condition and prove the results mentioned above.

\section{Disks, diagrams and critical points}

Disk. A disk is a pair $(z, r) \in \mathbb{R}^{3}$, where $z \in \mathbb{R}^{2}$ is the center of the disk and $\sqrt{r} \in \mathbb{C}$ its radius. Note that we also allow purely imaginary radii in which case the geometric intuition about disks is not helpful. We refer to $r$ also as the power of the disk.

In the following we are going to consider finite sets of disks. Very often these disks are not in general position. That is, we do not assume general position unless stated differently.

Power distance. The power distance of a point $x \in \mathbb{R}^{2}$ from a disk $(z, r)$ is $\pi(x)=\|x-z\|^{2}-r$.

Voronoi diagram. Given a set $B$ of disks. The Vorono $i$ cell of a disk $b_{i}$ under the power distance is given as

$$
V_{i}=\left\{x \in \mathbb{R}^{2}: \forall b_{j} \in B, \pi_{i}(x) \leq \pi_{j}(x)\right\}
$$

The sets $V_{i}$ are convex polygons or empty since the set of points that have the same power distance from two disks forms a straight line. Closed line segments shared by two Voronoi cells are called Voronoi edges and the points shared by three or more Voronoi cells are called Voronoi vertices. The term Voronoi object 
can denote either a Voronoi cell, edge or vertex. The Voronoi diagram of $B$ is the collection of Voronoi cells, edges and vertices. It defines a cell decomposition of $\mathbb{R}^{2}$.

Delaunay diagram. The Delaunay diagram of a set of disks $B$ is dual to the Voronoi diagram of $B$. The convex hull of three or more center points defines a Delaunay cell if the intersection of the corresponding Voronoi cells is not empty and there exists no superset of center points with the same property. Analogously, the convex hull of two center points defines a Delaunay edge if the intersection of their corresponding Voronoi cells is not empty. Every center point is called Delaunay vertex. The term Delaunay object can denote either a Delaunay cell, edge or vertex. The Delaunay diagram defines a decomposition of the convex hull of all center points. This decomposition is a triangulation if the disks are in general position.

Sometimes it is convenient to introduce an additional disk with center at infinity and with power 0 . This gives us a Voronoi vertex at infinity at the end of every unbounded Voronoi edge. One benefit of adding a disk at infinity is that it provides us with the following duality.

Duality. Let $B$ be a set of disks. This set gives rise to another set of disks, namely for every Delaunay cell its orthodisk, see [2] for the details. The new disks are centered at Voronoi vertices. The set $C$ exchanges Voronoi and Delaunay diagram.

Power height function. Let $B$ be a set of disks in $\mathbb{R}^{2}$. The power height function is given as

$$
h(x)=\min \left\{\pi_{i}(x): b_{i} \in B\right\} .
$$

Observe that the function $h$ is continuous. It is smooth everywhere besides at points which have the same power distance from two or more disks, i.e. at points that lie on the boundary of Voronoi cells.

Regular- and critical points. Let $B$ be a set of disks. The critical points of the power height function $h$ are the intersection points of Voronoi objects $V$ and their dual Delaunay object $\sigma$. The index of a critical point is the dimension of $\sigma$. All points which are not critical are called regular.

The power height function associated with a set of disks $B$ and the power height function associated with the dual set of disks $C$ have the same critical points. The index of a critical point in the dual is 2 minus its index in the primal, i.e. maxima and minima get exchanged.

\section{Disk induced flow}

Disk induced flow. Given a set $B$ of disks, the induced flow $\phi$ is given as follows: Since the Voronoi diagram of $B$ is a decomposition of the plane any point $x \in \mathbb{R}^{2}$ lies in some Voronoi object. Let $V$ be the lowest dimensional Voronoi object that contains $x$. Assume that $x$ is the intersection point of $V$ and its dual Delaunay object. In this case we set:

$$
\phi(t, x)=x, t \in[0, \infty)
$$


Otherwise let $\sigma$ be the dual Delaunay object of $V$ and $y=\operatorname{argmin}_{y^{\prime} \in \sigma}\left\|x-y^{\prime}\right\|$. Since $\sigma$ is convex there is only one such $y$. If $y$ also lies in a lower dimensional Delaunay object than $\sigma$ then we replace $V$ by the dual Voronoi object of the lowest dimensional Delaunay object. Let $R$ be the ray originating at $x$ and shooting in the direction $x-y$. Let $z$ be the first point on $R$ for which $\operatorname{argmin}_{y^{\prime} \in \tau}\left\|z-y^{\prime}\right\|$ is different from $y$ where $\tau$ denotes the dual Delaunay object of the lowest dimensional Voronoi object $z$ lies in. Note that such a $z$ need not exist in $\mathbb{R}^{2}$. In this case let $z$ be the point at infinity. We set:

$$
\phi(t, x)=x+t \frac{x-y}{\|x-y\|}, t \in[0,\|z-x\|]
$$

For $t>\|z-x\|$ the flow is given by property (2) in the definition of flow, i.e.

$$
\begin{aligned}
\phi(t, x) & =\phi(t-\|z-x\|+\|z-x\|, x) \\
& =\phi(t-\|z-x\|, \phi(\|z-x\|, x)) .
\end{aligned}
$$

It is not completely obvious but $\phi$ can be shown to be well defined on the whole of $[0, \infty) \times \mathbb{R}^{2}$. Furthermore, the following two properties of dynamical systems hold for disk induced flows,

(1) $\phi(0, x)=x$.

(2) $\phi(t+s, x)=\phi(t, \phi(s, x))$.

The following three observations are helpful to get a better understanding of disk induced flows and their their relationship to the power height function of the same set of disks.

(1) The fixpoints of $\phi$ are the critical points of the power height function.

(2) The orbits of $\phi$ are piecewise linear curves that are linear in Voronoi objects.

(3) The flow $\phi$ has no closed orbits.

Because of the first observation we want to refer to fixpoints of $\phi$ as minimum, saddle or maximum if the corresponding critical point of the power height function is a minimum, saddle or maximum, respectively.

Stable- and Unstable Manifolds. Given a disk induced flow $\phi$ in the plane. The stable manifold $S(x)$ of a fixpoint $x \in M$ contains all points that flow into $x$, i.e.

$$
S(x)=\left\{y \in \mathbb{R}^{2}: \lim _{t \rightarrow \infty} \phi_{y}(t)=x\right\} .
$$

The unstable manifold $U(x)$ of a fixpoint $x$ is a little bit more involved to define. Given a neighborhood $U$ of $x$ and let $V(U)$ be the set of all points which lie in an orbit that starts in $U$, i.e.

$$
V(U)=\left\{y \in \mathbb{R}^{2}: \exists z \in U, t \in[0, \infty) \text { s.t. } \phi_{z}(t)=y \text { or } \lim _{t \rightarrow \infty} \phi_{z}(t)=y\right\} .
$$

Then $U(x)$ is given as the intersection of all such sets $V(U)$. 

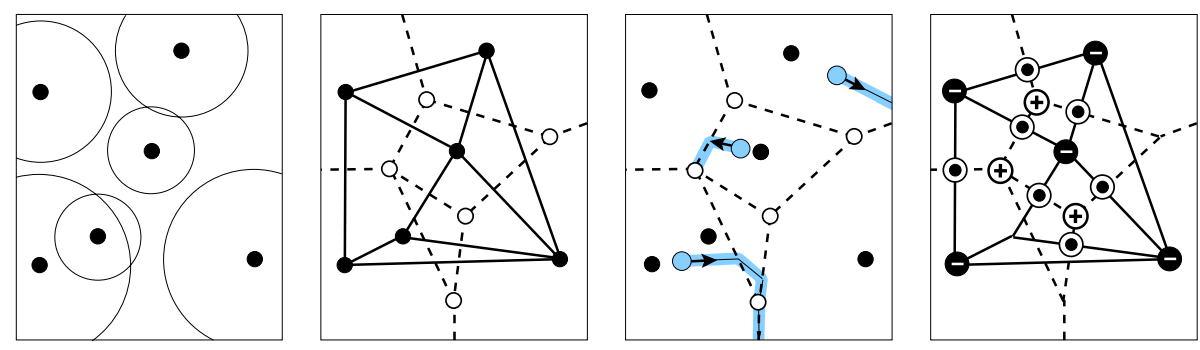

Fig. 1. A set of disk in the plane (on the left), its Voronoi- and Delaunay diagram (second from the left), some orbits of the flow induced by the disks (third form the left) and the minima $\ominus$, saddles $\odot$ and maxima $\oplus$ of this flow (on the right).

Instead of working directly with unstable manifolds of minima and stable manifolds of maxima we introduce Max- and Min regions which have nicer properties.

Max- and Min regions. Given a disk induced flow system with finitely many fixpoints. If $m$ is a maximum of this system we call the closure of the stable manifold $S(m)$ the Max region of $m$.

If $m$ is a minimum of a disk induced flow we call the closure of the interior of the unstable manifold $U(m)$ the Min region of $m$.
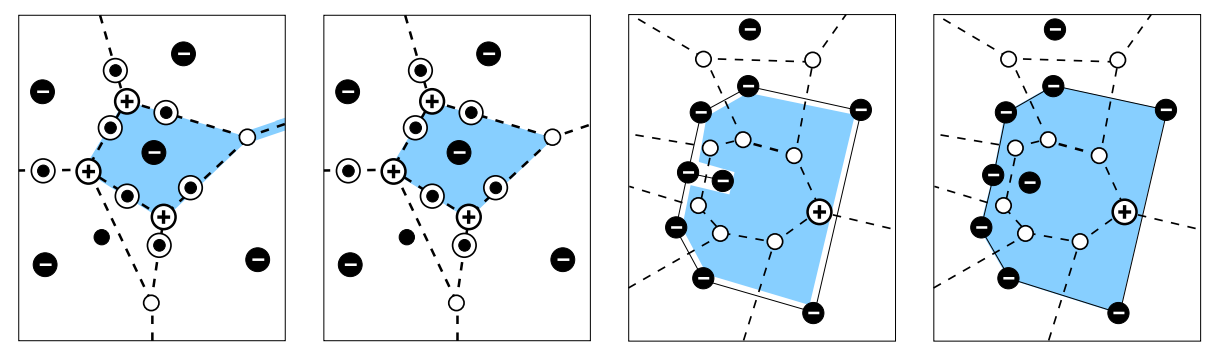

Fig. 2. An unstable manifold of a minimum $\ominus$ of the flow induced by the disks from Figure 1 (on the left) and its corresponding Min region (second from the left). The Min region is a true subset of the unstable manifold. A stable manifold of a maximum $\oplus$ of a different flow (second from the right) and its corresponding Max region (on the right). The Max region is a superset of the stable manifold.

Next we discuss stable and unstable manifolds of saddles which are a key tool to characterize and compute Max- and Min regions. Given a saddle $s$ be a saddle of $\phi$. We observe that

(1) The unstable manifold $U(s)$ of $s$ is a piecewise linear curve. 
(2) If the stable manifold $S(s)$ of $s$ does not contain a Voronoi vertex then closure $(S(s))$ is a piecewise linear curve.
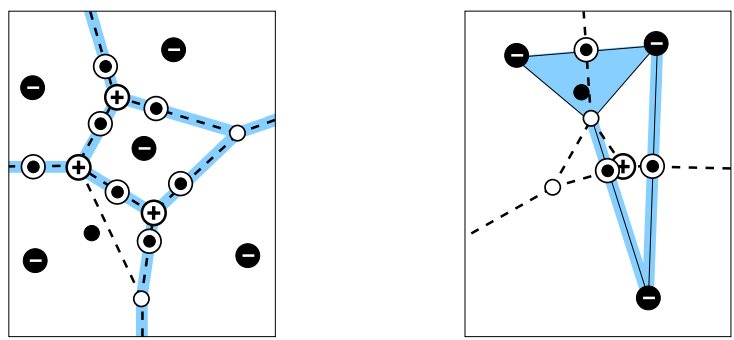

Fig. 3. The unstable manifolds of the saddles $\odot$ of the flow induced by the disks from Figure 1 (on the left). An example that shows that the stable manifolds of saddles $\odot$ need not be curves (on the right).

The second observation indicates that the stable manifolds of saddles can be more complicated to deal with than the unstable manifolds. In [5] we deal with this problems. Here we want to assume that the stable manifolds of all saddles do not contain any Voronoi vertex. This assumption is not really a restriction since it is automatically fulfilled under the strong duality condition which is the main topic of this paper.

The unstable manifolds of different saddles might join at some point or they might continue to infinity. The only points where two such unstable manifolds might join must lie on the boundaries of Voronoi cells, i.e. in the interior of Voronoi edges or at Voronoi vertices. If a point where two or more unstable manifolds of saddles join is not a maximum or saddle then we call it a Steiner vertex.

Unstable decomposition graph. Let $G$ be the following graph: Its vertex set consists of all maxima, saddles and Steiner vertices. Two vertices are connected by an edge if there exists an unstable manifold $U(s)$ of some saddle $s$ such that the two vertices are both contained in $U(s)$ and there is no other vertex in $U(s)$ between them. We add an additional vertex at infinity to the vertex set of $G$ and add edges that connect this point to all vertices of $G$ that flow under $\phi$ on a straight line to infinity. We refer to $G$ as the unstable decomposition graph associated with a disk induced flow. Let $S$ be the set of saddles of $\phi$ and $U(S)=\bigcup_{s \in S} U(s)$. We refer to $U(S)$ as the geometric realization of $G$.

Stable decomposition graph. Let $G$ be the following graph: Its vertex set consists of all minima, saddles and joins. Two vertices are connected by an edge if there exists a stable manifold $S(s)$ of some saddle $s$ such that the two vertices are both contained in closure $(S(s))$ and there is no other vertex on $S(s)$ between them. We have to take the closure of the stable manifolds because otherwise the minima would not be contained in them. We refer to $G$ as the 
stable decomposition graph associated with a disk induced flow. Let $S$ be the set of saddles of $\phi$ and $S(S)=\bigcup_{s \in S} \operatorname{closure}(S(s))$. We refer to $S(S)$ as the geometric realization of $G$.

Theorem 1. The following is true.

(1) The maximum complexities of the geometric realizations of the stable- and unstable decomposition graph of $n$ disks are both $\Theta\left(n^{2}\right)$ vertices, $\Theta\left(n^{2}\right)$ straight line segments and $\Theta(n)$ regions.

(2) The worst case algorithmic complexities of the stable- and unstable decomposition graph of $n$ disks are both $\Theta\left(n^{2}\right)$.

In [4] and [5] we establish a connection of the unstable decomposition graph and the Min regions of the same set of disks and a connection of the stable decomposition graph and the Max regions.

Theorem 2. The following is true:

(1) The regions of the unstable decomposition graph of a set of disks are exactly the Min regions of the flow associated with these disks.

(2) The regions of the stable decomposition graph of a set of disks are exactly the Max regions of the flow associated with these disks.

\section{Strong duality}

Strong duality condition. A set $B$ of disks in $\mathbb{R}^{2}$ obeys the strong duality condition if the center of each disk in $B$ is contained in its dual Voronoi cell, i.e. every disk center is a minimum of the flow induced by $B$.

Observe that the strong duality condition is always obeyed by a set of points, i.e. a set of disks that all have radius zero.

The Gabriel graph of a set of disks is a well studied object that has applications in many areas. It turns out that the unstable decomposition graph is a Gabriel graph under the strong duality condition.

Gabriel graph. Given a set $B$ of disks in the plane. The Gabriel graph of $G$ is given as follows: Its vertices are the centers of the disks in $B$ and its edges are given by Delaunay edges that intersect their dual Voronoi edge. The edges of the Gabriel graph are called Gabriel edges. From the Definition of critical points we know that there is a one to one correspondence between Gabriel edges and saddles of $\phi_{B}$.

Theorem 3. The following is true.

(1) The maximum geometric complexity of a Gabriel graph of $n$ disks is $\Theta(n)$ vertices, $\Theta(n)$ edges and $\Theta(n)$ regions.

(2) The worst case algorithmic complexity, i.e. the number of steps it takes to compute the Gabriel graph of $n$ disks, is $\Theta(n \log n)$. 
Theorem 4. Given a set $B$ of disks that obeys the strong duality condition plus one additional disk at infinity. Let $C$ be the set of dual disks and $\phi_{B}$ and $\phi_{C}$ be the flows induced by $B$ and $C$, respectively. The following is true:

(1) Every disk center of $B$ is a minimum of $\phi_{B}$ and a maximum of $\phi_{C}$.

(2) The geometric realizations of the unstable decomposition graph associated with $\phi_{C}$ and the stable decomposition graph associated with $\phi_{B}$ are both the Gabriel graph of $B$.

Proof. (1) This follows just from the definition of critical points. (2) Every saddle of $\phi_{B}$ is by definition also a saddle of $\phi_{C}$ and vice versa. The Delaunay edge (with respect to $B$ ) that contains some saddle $s$ is a Gabriel edge by the definitions of saddles and Gabriel edges, respectively. This Gabriel edge is a Voronoi edge with respect to $C$ and the endpoints of this edge are minima of $\phi_{B}$ and maxima of $\phi_{C}$ by the strong duality condition and the definition of duality. That is, $s$ is connected by this Gabriel edge to two minima with respect to $B$ and to two maxima with respect to $C$. Hence this Gabriel edge is both $U(s)$ (with respect to $C$ ) and $S(s)$ (with respect to $B$ ). This implies that the geometric realizations of the unstable decomposition graph associated with $\phi_{C}$ and the stable decomposition graph associated with $\phi_{B}$ are the Gabriel graph of $B$.

Corollary 1. The following is true:

(1) The Max regions of $\phi_{B}$ are exactly the bounded Min regions of $\phi_{C}$.

(2) The Max regions of $\phi_{B}$ are a set of contiguous Delaunay cells of B separated by Gabriel edges.

(3) The maximum complexities of the geometric realization of the stable decomposition graph associated with $\phi_{B}$ and the geometric realization of the unstable decomposition graph associated with $\phi_{C}$ are both $\Theta(n)$ vertices, $\Theta(n)$ edges and $\Theta(n)$ regions.

(4) The worst case algorithmic complexities of the geometric realization of the stable decomposition graph associated with $\phi_{B}$ and the geometric realization of the unstable decomposition graph associated with $\phi_{C}$ are both $\Theta(n \log n)$.
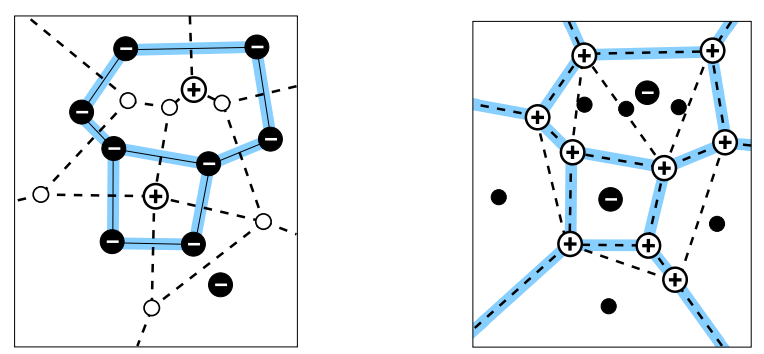

Fig. 4. Max regions of maxima $\oplus$ in the primal (on the left) are bounded Min regions of minima $\ominus$ in the dual (on the right) under the strong duality condition. Note that here the boundaries of these regions are emphasized. 
The analogous result to the first observation for the bounded Min regions of $\phi_{B}$ and the Max regions of $\phi_{c}$ does not hold as can be seen easily from examples. Note that the the set $C$ of dual disks need not fulfill the strong duality condition even if $B$ does.

Under the strong duality condition we have a simpler characterization of the Min regions of $\phi_{B}$.

Theorem 5. Let $B$ a set of disks that obeys the strong duality condition and $\phi$ the flow induced by $B$. The Min regions of $\phi$ are the Voronoi cells of $B$.

Proof. Let $z$ be the center of a disk in $B$ and $V$ its dual Voronoi region. By the strong duality condition $z$ lies in the interior of $V$ which implies that $z$ is a minimum of $\phi$. From the definition of $\phi$ we have that the interior of $V$ is a subset of the Min region $M$ of $z$. Furthermore, $V$ is a subset of $M$ since Min regions are always closed. Now assume that $V$ is a true subset of $M$. The difference $M-V$ has to contain an open subset $U$ of $\mathbb{R}^{2}$, because by definition Min regions are the closure of the interior of some subset of $\mathbb{R}^{2}$. The set $U$ must have a non empty intersection with the interior of some Voronoi cell $V^{\prime}$ different from $V$. The interior of $V^{\prime}$ is part of the Min region of its dual Delaunay vertex $m^{\prime}$. Let $x \in U \cap V^{\prime}$. By the definition of $\phi$ the closure of the set $\left\{y \in \mathbb{R}^{2}: \exists t \in\right.$ $[0, \infty)$ s.t. $\left.\phi_{y}(t)=x\right\}$ is the line segment that connects $m^{\prime}$ with $x$. Thus $x$ belongs neither to the unstable nor to the Min region of $m$. That is a contradiction to our assumption. Hence $M=V$.

Corollary 2. The following is true:

(1) The maximum complexities of the geometric realization of the unstable decomposition graph associated with $\phi_{B}$ is $\Theta(n)$ vertices, $\Theta(n)$ edges and $\Theta(n)$ regions.

(2) The worst case algorithmic complexity of the geometric realization of the unstable decomposition graph associated with $\phi_{B}$ is $\Theta(n \log n)$.

The strong duality condition allows to define a discrete flow, i.e. a discrete dynamical system on the set of triangles of the Delaunay triangulation [3]. In the following we assume that the disks are in general position, i.e. the Delaunay diagram is actually a triangulation.

Given a triangle in the Delaunay triangulation of a set of disks. We associate every edge of the triangle with the halfspace that intersects the triangle only in this edge. We call the wedge in the intersection of two such halfspaces that does not contain the triangle a Delaunay wedge.

Lemma 1. Let $B$ be a set of disks that obeys the strong duality condition. Then no Voronoi vertex is contained in a Delaunay wedge of its dual Delaunay triangle.

Proof. Assume the contrary, i.e. there exists a Voronoi vertex $v$ that is contained in a Delaunay wedge of its dual Delaunay triangle $\sigma$. Let $x$ be the Delaunay vertex that is incident to the two edges of $\sigma$ whose associated halfspaces define the wedge that contains $x$. By construction $x$ cannot be contained in the wedge 
centered at $v$ and bounded by the two Voronoi edges dual to the two Delaunay edges incident to $x$. That is, $x$ is not contained in its dual Voronoi cell. That contradicts the strong duality condition.

Triangle flow. Given a set $B$ of disks that obeys the strong duality condition. Let $\mathcal{D}$ denote the set of all Delaunay triangles in the Delaunay triangulation of $B$ together with an abstract triangle inf at infinity. The flow on $\mathcal{D}$ is a function $\Phi: \mathbb{N}_{0} \times \mathcal{D} \rightarrow \mathcal{D}$ which satisfies

(1) $\Phi(0, \sigma)=\sigma$ for every $\sigma \in \mathcal{D}$.

(2) $\Phi(n+m, \sigma)=\Phi(n, \Phi(m, \sigma))$ for every $\sigma \in \mathcal{D}$.

That is, $\Phi$ is defined recursively. The recursion has to be anchored by defining $\Phi(1, \sigma)$. We set $\Phi(1, \sigma)=\sigma$ if $\sigma=$ inf or the dual Voronoi vertex $v$ of $\sigma$ is contained in $\sigma$. Otherwise $v$ is contained in exactly one of the halfspaces associated with the edges of $\sigma$. If there exists a second Delaunay triangle $\sigma^{\prime}$ incident to the edge that corresponds to the halfspace that contains $v$ we set $\Phi(1, \sigma)=\sigma^{\prime}$. Otherwise we set $\Phi(1, \sigma)=$ inf.

We find directly from the definitions that the fixpoints besides inf of a triangle flow are exactly the dual triangles of the maxima of the continuous flow induced by the same set of disks.

Theorem 6. Let $B$ be a set of disks that obeys the strong duality condition and $\phi$ the flow induced by $B$. Let $m$ be a maximum of $\phi$ and $\sigma$ its dual Delaunay triangle. Then the Max region of $m$ is the following set

$$
\left\{x \in \sigma^{\prime} \in \mathcal{D}: \exists n \geq 0 \text { such that } \Phi\left(n, \sigma^{\prime}\right)=\sigma\right\},
$$

where $\Phi$ is the triangle flow on the triangle set $\mathcal{D}$ of the Delaunay triangulation of $B$.

Proof. First observe that the flow $\Phi$ has to be acyclic, because the power of the orthodisks of the Delaunay triangles increases in every time step of the flow $\Phi$.

By construction the flow $\Phi$ cannot pass any Gabriel edge. Every region in the Gabriel graph of $B$ contains exactly one maximum of $\phi$, i.e. one fixpoint of $\Phi$. Since $\Phi$ is acyclic all triangles in a region of the Gabriel graph have to flow into this unique fixpoint. From Corollary 1(2) we know that all points in this region flow into $m$ under the flow $\phi$.

\section{References}

1. F. Aurenhammer and R. Klein. Voronoi Diagrams. In Handbook of Computational Geometry, J.-R. Sack and J. Urrutia (eds.), pp. 201-290, Elsevier (2000)

2. S.W. Cheng, H. Edelsbrunner, P. Fu and K.P. Lam. Design and Analysis of Planar Shape Deformation. Proc. 14th Symp. Comp. Geom. pp. 29-38, (1998)

3. H. Edelsbrunner, M. A. Facello and J. Liang. On the definition and the construction of pockets in macromolecules. Discrete Apl. Math. 88, pp. 83-102, (1998)

4. J. Giesen and M. John. Dynamical Systems from Disks in the Plane. Manuscript (2001)

5. J. Giesen and M. John. A new Diagram from Disks in the Plane. Accepted for Proc. 19th Int. Symp. Theo. Aspects of Comp. Sc. (2002) 